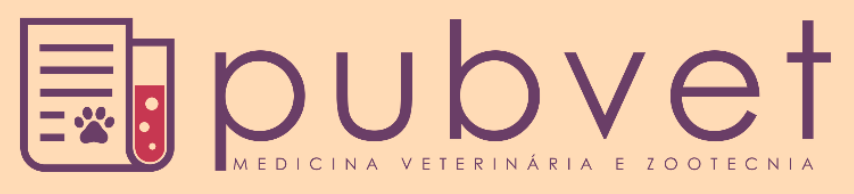

https://doi.org/10.31533/pubvet.v14n11a681.1-4

\title{
Dieta natural no tratamento de cão acometido com recorrentes urólitos de oxalato de cálcio: Relato de caso
}

\author{
Anna Clara Leite ${ }^{1}$, Aline da Conceição Almeida ${ }^{1}$, Alexandre Herculano Borges de Araújo ${ }^{10}$, \\ Erica Beatriz Schultz ${ }^{* \bullet}$, Brennda Paula Gonçalves de Araújo ${ }^{1}$, Silvia Vitória Santos de \\ Carvalho Araújo ${ }^{1}{ }^{\ominus}$, Rosana Colatino Soares Reis ${ }^{1 \odot}$
}

${ }^{I}$ Universidade Federal Rural do Rio de Janeiro, Instituto de Zootecnia, Seropédica, Rio de Janeiro.

*Autor para correspondência: ericabeatrizschultz@gmail.com

\begin{abstract}
Resumo: Os urólitos, que causam as urolitíases, são um exemplo dos efeitos de deficiências ou excessos de nutrientes e minerais na alimentação. Com intuito de inibir o crescimento e reincidência de urolitíases uma alternativa é a adoção da alimentação natural para cães. A alimentação natural é aliada na prevenção dos cálculos devido à possibilidade de se manipular um alimento específico para cada indivíduo, respeitando as particularidades e limitações impostas pela urolitíase. Com base nesta premissa, objetivouse utilizar a alimentação natural como tratamento e prevenção no estudo de caso de um cão com urólitos de oxalato reincidente, cujo tratamento inicial com ração comercial específica não fora satisfatório. A formulação da dieta natural foi realizada com base nas quantidades proteína, carboidratos, óleo e sódio, a fim de alcalinizar o $\mathrm{pH}$ urinário de modo e evitar a formação de novos cálculos. A dieta natural proporcionou melhora no caso do animal, que apesar de não ter sido no pH urinário, não houve a formação de novos cristais.
\end{abstract}

Palavras chave: cristais, dieta, urolitíase

\section{Natural diet in treatment of dog affected with recurrent calcium oxalate uroliths: Case report}

\begin{abstract}
Uroliths, which cause urolithiasis, is an example of the effects of nutrients and minerals deficiency or excess in the diet. In order to cease the growth and recurrence of urolithiasis, an alternative is the adoption of natural food. Natural food is related to the stones prevention due to the possibility of manipulating a specific feed for each individual, regarding the particularities and limitations imposed by urolithiasis. Based on this premise, the objective was to use natural food as a treatment and prevention in the case report of a dog with recurrent oxalate uroliths, whose initial treatment with specific commercial feed was not satisfactory. The formulation of the natural diet was performed on the amounts of protein, carbohydrates, oil and sodium, in order to alkalinize the urinary $\mathrm{pH}$, and slow the formation of new stones. The natural diet showed improvement in the case of the animal, which despite having no change in urinary $\mathrm{pH}$, did not have the formation of new crystals.
\end{abstract}

Keywords: crystals, diet, urolithiasis

\section{Introdução}

Os urólitos são sedimentos sólidos formados a partir do acúmulo de precipitados resultante de falhas na excreção de metabólitos pela urina, gerando assim os cristais (Monferdini \& Oliveira, 2009), que podem ser formados desde a pelve renal até a uretra canina (Muniz Neta \& Munhoz, 2008; Oyafuso, 2008; Rick et al., 2017). Estes são classificações de acordo com a composição dos cristais, como 
exemplo, estruvita, fosfato de cálcio e oxalato de cálcio, sendo imprescindível o conhecimento da composição para estabelecer tratamento (Kaufmann et al., 2011).

A formação dos urólitos de oxalato de cálcio acontece em faixas de $\mathrm{pH}$ inferior a 6,5 ocorrendo supersaturação da urina com cálcio e oxalato e posterior absorção intestinal de cálcio (Monferdini \& Oliveira, 2009). Dentre os fatores que podem contribuir para a formação do cálculo de oxalato de cálcio estão, o racial, sendo comum, por exemplo, em Lulu, Shih Tzu, Yorkshire Terrier, desbalanço nutricional, a hipercalcemia e a administração de medicamentos calciuréticos, como glicocorticoides e furosemida (Elliot, 2003; Koehler et al., 2009).

Para este tipo de urólito não há nenhum método de dissolução, portanto, o protocolo a ser utilizado é a remoção cirúrgica (Okafor et al., 2014). Entretanto, segundo Sturgess (2009), o manejo nutricional é crucial para minimizar a ocorrência desse tipo de cálculo. A dieta preventiva busca alcalinizar a urina e minimizar o consumo de vitamina $\mathrm{C}$, o que favorece a hiperoxalúria, além do controle de vitamina $\mathrm{D}$ e cálcio, pois, de acordo com (Dijcker et al., 2011) a ingestão excessiva de cálcio e vitamina D, favorece os riscos de aparecimento de cálculos de oxalato de cálcio.

Neste contexto, a formulação de dietas naturais para pets tem ganhado espaço pela versatilidade, possibilitando ao nutricionista adequar a alimentação ao perfil nutricional de cada indivíduo, levando em consideração às diferentes fases fisiológicas e estados fisiopatológicos (Halfen et al., 2017). Portanto, objetivou-se o tratamento e prevenção de novos cristais utilizando dieta natural específica para cão com casos reincidentes de urólitos de oxalato de cálcio.

\section{Relato de caso}

O estudo foi realizado com um cão do sexo masculino, raça York Shire Terrier, com idade de 10 anos, vacinado e vermifugado. Devido aos sintomas de apatia e hematúria, o animal foi atendido em clínica especializada onde foram realizados exames físicos e ultrassonográficos. À palpação, durante o exame físico, verificou-se sensibilidade abdominal e presença de massa endurecida na região ventrocaudal do abdome (bexiga), suspeitou-se, então, de cálculo vesical. Contudo, os médicos veterinários diagnosticaram a presença de cristais, identificados posteriormente como de oxalato de cálcio na vesícula urinária. Como forma de tratamento elegeu-se o procedimento cirúrgico, que fora realizada no dia 03/05/2019.

Após a retirada dos cristais a alimentação foi exclusiva com ração comercial do tipo urinária. Através do acompanhamento clínico do animal foi observado acúmulo de microcristais na uretra levando à segunda cirurgia, realizada no dia 18/09/2019. Devido ao tamanho dos cristais, houve a obstrução da uretra do animal, necessitando se realizar uma uretrostomia (Figura 1). Foram retirados dois cálculos com 1,8 e 1,9 cm de comprimento, além de vários urólitos fragmentados (Figura 2, Figura 3).

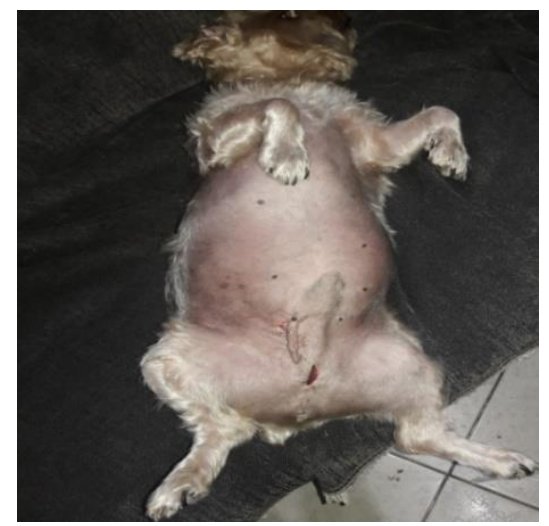

Figura 1. Animal após a uretrostomia

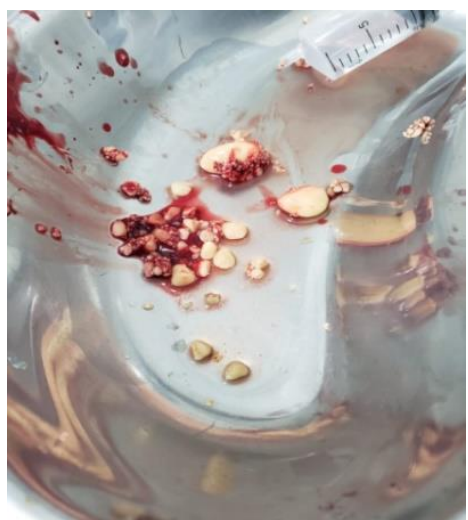

Figura 2. Fragmentos de cálculos retirados do animal

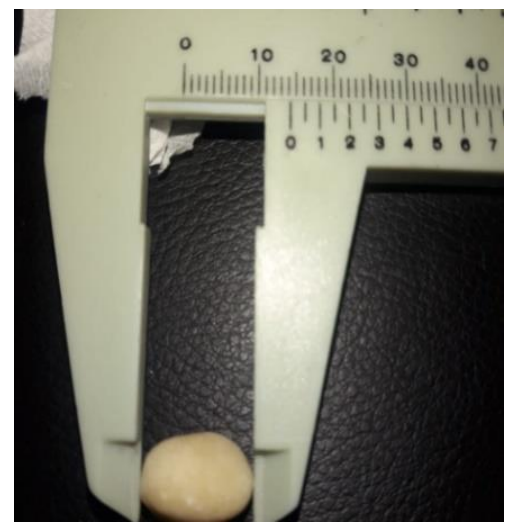

Figura 3. Cálculo retirado do animal

Devido ao diagnóstico e a reincidência de cálculos mesmo com a ração específica, no dia 25/09/2019, foi proposto o fornecimento de dieta natural com objetivo de alcalinizar o $\mathrm{pH}$ urinário, equilibrando os ácidos e bases presentes na urina, de modo a diminuir a acidez e a formação de novos cálculos. 
A dieta proposta pela nutricionista teve como base os alimentos divididos nas seguintes classes: proteínas, carboidratos coloridos, carboidratos verdes, óleo e sódio (Tabela 1).

Tabela 1. Quantidade e composição nutricional da dieta

\begin{tabular}{lccccc}
\hline Quantidade fornecida & Fontes Proteína & CHO brancos & CHO coloridos & CHO verdes & Óleo \\
\hline $350 \mathrm{~g}$ & $50 \mathrm{~g}$ & $70 \mathrm{~g}$ & $115 \mathrm{~g}$ & $115 \mathrm{~g}$ & $6 \mathrm{~g}$ \\
\hline
\end{tabular}

CHO: carboidratos.

A quantidade de sódio indicada foi de acordo com a de água utilizada para o cozimento dos alimentos. A cada 1 litro de água no cozimento, foi recomendada a proporção de uma colher de chá de sal, isso equivale a aproximadamente $10 \mathrm{~g}$ em 1 litro de água, concentração muito próxima da solução fisiológica.

A partir disso, o tutor teve uma lista de opções de alimentos referentes a cada classe citada, podendo variar a alimentação do animal a cada semana, respeitando os limites de quantidade estabelecidos.

Foi proibido o consumo de talos, sementes e cascas, devido à maior quantidade de minerais presentes nesses alimentos, o que poderia contribuir para a formação de novos cristais.

O tutor foi o responsável por preparar o alimento. A cada semana foi escolhido um alimento de cada classe (proteínas, carboidratos brancos, coloridos e verdes), e no preparo todos os ingredientes eram cozidos na mesma panela. Quando pronto, o objetivo era um alimento com a textura al dente.

A partir da dieta estabelecida, o animal consumiu a quantidade diária de $350 \mathrm{~g}$, dividida em duas refeições de $175 \mathrm{~g}$ cada respeitando o intervalo de 12 horas entre as ingestas. Foi realizada suplementação com vitamina D (1 gota/kgPV), cujo objetivo foi aumentar a fixação de cálcio, diminuindo a concentração na urina.

Após um mês e sete dias com a nova dieta natural, foram realizados novos exames laboratoriais para acompanhamento do caso. Os novos exames de urina, mostraram que o $\mathrm{pH}$ urinário se manteve estável, e que não houve o aparecimento de novos cristais (Tabela 2). Além disso, os parâmetros como cor, aspecto e densidade da urina se mostraram dentro dos resultados referência.

Tabela 2. pH urinário antes e pós alimentação natural

\begin{tabular}{cc}
\hline $\mathrm{pH}$ antes da cirurgia $(14 / 09 / 2019)^{*}$ & $\mathrm{pH}$ pós alimentação natural $(02 / 11 / 2019)^{*}$ \\
\hline 6,5 & 6,0 \\
\hline
\end{tabular}

*Dados fornecidos pelo centro de apoio e diagnóstico veterinário $\left(\mathrm{CAD}^{\circledR}\right)$.

\section{Discussão}

A formação e reincidência de cálculos renais no cão estudado estão relacionadas ao fator racial e nutricional. De acordo com Monferdini \& Oliveira (2009), as principais causas das formações de cristais e urólitos podem ser atribuídas à diminuição na frequência da micção, somadas a concentrações elevadas de solutos (principalmente minerais), que supersaturam a urina. Por isso, as chances de ocorrência de urolitíase são maiores em cães de raças pequenas e gatos que são alimentados com rações secas, devido ao baixo teor de umidade que os leva a produzir menor quantidade de urina e consequentemente a menor frequência de micção. Logo, além de o animal em estudo estar dentro do grupo de raças pequenas, a troca pela ração comercial específica não trouxe benefícios na prevenção de aparecimento de urolitiase, pois, permaneceu com alimento de baixa umidade.

A urina é o principal e mais eficiente meio utilizado pelo organismo para eliminação de resíduos do metabolismo proteico (ureia, amônia e creatinina), além de água e minerais. $\mathrm{O}$ pH urinário é resultado da manutenção homeostática do equilíbrio ácido-básico (Chew et al., 2012).

A dieta tem papel fundamental no aparecimento e/ou prevenção de urolitíase. Segundo Carciofi (2008), a dieta interfere diretamente no $\mathrm{pH}$, na densidade específica e no volume urinário.

Um ponto forte na manipulação da dieta para tratamento e prevenção da urolitíase é a ingestão de água. $\mathrm{O}$ aumento no consumo de água auxilia na diluição da urina, inibindo a formação e crescimento de cálculos, independente da natureza. 
A ingestão de água pode ser estimulada com o fornecimento de dietas com teor de umidade elevada, como por exemplo, na alimentação natural. Dessa forma, aumenta-se a ingestão de água total e consequentemente aumenta-se o volume de urina, reduzindo a concentração da mesma (Dijcker et al., 2011). Fato que explica a não formação de novos cálculos pelo animal embora a manutenção do pH urinário ao contrastar com o pH quando em consumo de ração comercial.

\section{Conclusão}

A dieta natural apresentou eficácia no tratamento e prevenção do cão. Recomendou se o acompanhamento contínuo do cão com o médico veterinário e zootecnista visando o monitoramento da formação ou não de novos cálculos. A análise do cálculo foi fundamental para a correta elaboração da dieta, bem como avaliar possíveis reicidivas.

\section{Referências bibliográficas}

Carciofi, A. C. (2008). Manejo nutricional do cão e do gato hospitalizado. In UNESP (Ed.), Apontamentos teóricos das disciplinas de Clínica das Doenças Carenciais, Endócrinas e Metabólicas e de Nutrição e Alimentação de Cães e Gatos. Universsidade de São Paulo.

Chew, D. J., Dibartola, S. P., \& Schnck, P. A. (2012). Urologia e nefrologia do cão e do gato. SaundersEnsevier.

Dijcker, J. C., Plantinga, E. A., Van Baal, J., \& Hendriks, W. H. (2011). Influence of nutrition on feline calcium oxalate urolithiasis with emphasis on endogenous oxalate synthesis. Nutrition Research Reviews, 24(1), 96-110. DOI: https://doi.org/10.1017/s0954422410000351

Elliot, D. A. (2003). How I treat... the dog with calcium oxalate urolithiasis. Revista Waltham Focus, 2-3.

Halfen, D. P., Oba, P. M., Duarte, C. N., Santos, J. P. F., Vendramini, T. H. A., Sucupira, M. C. A., Carciofi, A. C., \& Brunetto, M. (2017). Tutores de cães consideram a dieta caseira como adequada, mas alteram as fórmulas prescritas. Pesquisa Veterinária Brasileira, 37(12), 1453-1459. DOI: https://doi.org/10.1590/s0100-736x2017001200015

Kaufmann, C., Neves, R. C., \& Habermann, J. C. A. (2011). Doença do trato urinário inferior dos felinos. Anuário Da Produção Científica Dos Cursos de Pós-Graduação, 4(4), 193-214.

Koehler, L. A., Osborne, C. A., Buettner, M. T., Lulich, J. P., \& Behnke, R. (2009). Canine uroliths: frequently asked questions and their answers. Veterinary Clinics of North America: Small Animal Practice, 39(1), 161-181. DOI: https://doi.org/10.1016/j.cvsm.2008.09.007

Monferdini, R. P., \& Oliveira, J. (2009). Manejo nutricional para cães e gatos com urolitíase-Revisão bibliográfica. Acta Veterinaria Brasilica, 3(1), 1-4.

Muniz Neta, E. dSouza, \& Munhoz, A. D. (2008). Urolitíase em cães e gatos: uma revisão. MEDVEP. Revista Científica de Medicina Veterinária, 6(17), 24-34.

Okafor, C. C., Lefebvre, S. L., Pearl, D. L., Yang, M., Wang, M., Blois, S. L., Lund, E. M., \& Dewey, C. E. (2014). Risk factors associated with calcium oxalate urolithiasis in dogs evaluated at general care veterinary hospitals in the United States. Preventive Veterinary Medicine, 115(3-4), 217-228. DOI: https://doi.org/10.1016/j.prevetmed.2014.04.006

Oyafuso, M. K. (2008). Estudo retrospectivo e prospectivo da urolitíase em cães. Universidade de São Paulo. DOI: https://doi.org/10.11606/d.10.2008.tde-12122008-104152

Rick, G. W., Conrad, M. L. H., Vargas, R. M., Machado, R. Z., Lang, P. C., Serafini, G. M. C., \& Bones, V. C. (2017). Urolitíase em cães e gatos. PUBVET, 11, 646-743. DOI: https://doi.org/10.22256/pubvet.v11n7.705-714

Sturgess, K. (2009). Dietary management of canine urolithiasis. In Practice, 31(7), 306-312. DOI: https://doi.org/10.1136/inpract.31.7.306

Recebido: 26 de abril, 2020. Aprovado: 3 de junho, 2010.

Disponível online: 23 outubro, 2020.
Licenciamento: Este artigo é publicado na modalidade Acesso Aberto sob a licença Creative Commons Atribuição 4.0 (CC-BY 4.0), a qual permite uso irrestrito, distribuição, reprodução em qualquer meio, desde que o autor e a fonte sejam devidamente creditados 